\title{
Mark the transition: chromatin modifications and cell fate decision
}

\author{
Qiang $\mathrm{Wu}^{1}$, Huck-Hui $\mathrm{Ng}^{1,2,3,4,5}$
}

${ }^{1}$ Department of Biochemistry, Yong Loo Lin School of Medicine, National University of Singapore, 8 Medical Drive, Singapore 117597; ${ }^{2}$ Gene Regulation Laboratory, Genome Institute of Singapore, 60 Biopolis Street, Singapore 138672; ${ }^{3}$ NUS Graduate School for Integrative Sciences and Engineering, National University of Singapore, Singapore 117456; ${ }^{4}$ Department of Biological Sciences, National University of Singapore, Singapore 117597; 5 School of Biological Sciences, Nanyang Technological University, Singapore 639798

Cell Research (2011) 21:1388-1390. doi:10.1038/cr.2011.154; published online 20 September 2011

With their unique features of selfrenewal and pluripotency, human embryonic stem (hES) cells are considered to be a nearly unlimited resource for research and clinical applications [1]. Accordingly, the transcriptional network specifying and governing human ES cell identity has been extensively studied. OCT4, NANOG and SOX2 form a core transcriptional network that regulates itself as well as a number of target genes [2]. This transcriptional network acts together with signaling pathways to maintain ES cell identity [3]. Moreover, the last decade has seen tremendous advances in understanding the epigenetic mechanisms underlying ES cell self-renewal and pluripotency. It is believed that the so-called "bivalent" histone modification (promoters of many developmental genes that co-harbor the active $\mathrm{H} 3 \mathrm{~K} 4 \mathrm{me} 3$ and the silencing $\mathrm{H} 3 \mathrm{~K} 27$ me3 marks) is required for undifferentiated ES cells to be poised to quickly differentiate into certain lineages [2]. Besides H3K4me3, recent studies have indicated that histone $\mathrm{H} 3 \mathrm{~K} 4 \mathrm{me} 1$ and $\mathrm{H} 3 \mathrm{~K} 27 \mathrm{ac}$ are the

Correspondence: Qiang Wu $\mathrm{Wu}^{\mathrm{a}}$, Huck-Hui $\mathrm{Ng}^{\mathrm{b}}$

aE-mail: bchwq@nus.edu.sg

bE-mail: nghh@gis.a-star.edu.sg marks of active regulatory regions, such as promoters and enhancers, in the mammalian genome [4]. Given that transcription factors, cofactors and chromatin modifications are enriched in these regulatory regions, it is important to investigate the role of $\mathrm{H} 3 \mathrm{~K} 4 \mathrm{me} 1$ and $\mathrm{H} 3 \mathrm{~K} 27 \mathrm{ac}$ in regulation of ES cell pluripotency and differentiation.

In this issue of Cell Research, Hawkins et al. addressed many aspects of this question [5]. The authors first induced $\mathrm{hES}$ cells to differentiate into a mixture of mesendodermal and trophectodermal cells (denoted as DFC). They then mapped the profiles of $\mathrm{H} 3 \mathrm{~K} 4 \mathrm{me} 1$, H3K4me3, H3K27me3 and H3K27ac by ChIP-on-chip in both hESCs and DFCs. Interestingly, the authors discovered that a number of promoters display a dynamic switch between H3K27me3 and $\mathrm{H} 3 \mathrm{~K} 27 \mathrm{ac}$ while the presence of $\mathrm{H} 3 \mathrm{~K} 4 \mathrm{me} 3$ is not correlated with cell fate decision. This finding led the authors to further define the switch upon differentiation. They found that genes active in hESCs but suppressed in DFCs generally display $\mathrm{H} 3 \mathrm{~K} 27 \mathrm{ac}$ loss and H3K27me3 gain after cell commitment. These genes include key transcription factors and important WNT signaling molecules, which are promoters of ES cell pluripotency. On the other hand, genes suppressed in hESCs but active in DFCs exhibit H3K27ac gain and H3K27me3 loss after differentiation. Genes from the latter category, such as $H A N D 1$, were actively expressed following differentiation. With these results, the author proposed that since monovalent $\mathrm{H} 3 \mathrm{~K} 4 \mathrm{me} 3$ genes are not always expressed, it would be more expedient to measure changes in $\mathrm{H} 3 \mathrm{~K} 27 \mathrm{ac}$ and $\mathrm{H} 3 \mathrm{~K} 27 \mathrm{me} 3$ instead of monitoring changes in bivalent genes to determine the cell fate transition.

After examining the switch of $\mathrm{H} 3 \mathrm{~K} 27 \mathrm{ac}$ and $\mathrm{H} 3 \mathrm{~K} 27 \mathrm{me} 3$ in promoters, Hawkins et al. investigated the role of enhancers in hESCs. To this end, the authors used a computational method based on $\mathrm{H} 3 \mathrm{~K} 4 \mathrm{me} 1$ and $\mathrm{H} 3 \mathrm{~K} 4 \mathrm{me} 3$ patterns since both are hallmarks of active genes, consequently, the authors identified nearly 29000 enhancers in hESCs and over 33000 enhancers in DFCs. These enhancers have several features: (1) they are mainly distal to transcription start sites (TSS). Nearly $50 \%$ of these enhancers are found in intergenic regions and over $40 \%$ in intragenic regions. (2) Many enhancers are near important pluripotency regulators such as FOXD3. (3) These enhancers are often clustered. To further characterize these putative enhancers, the authors then pre- 


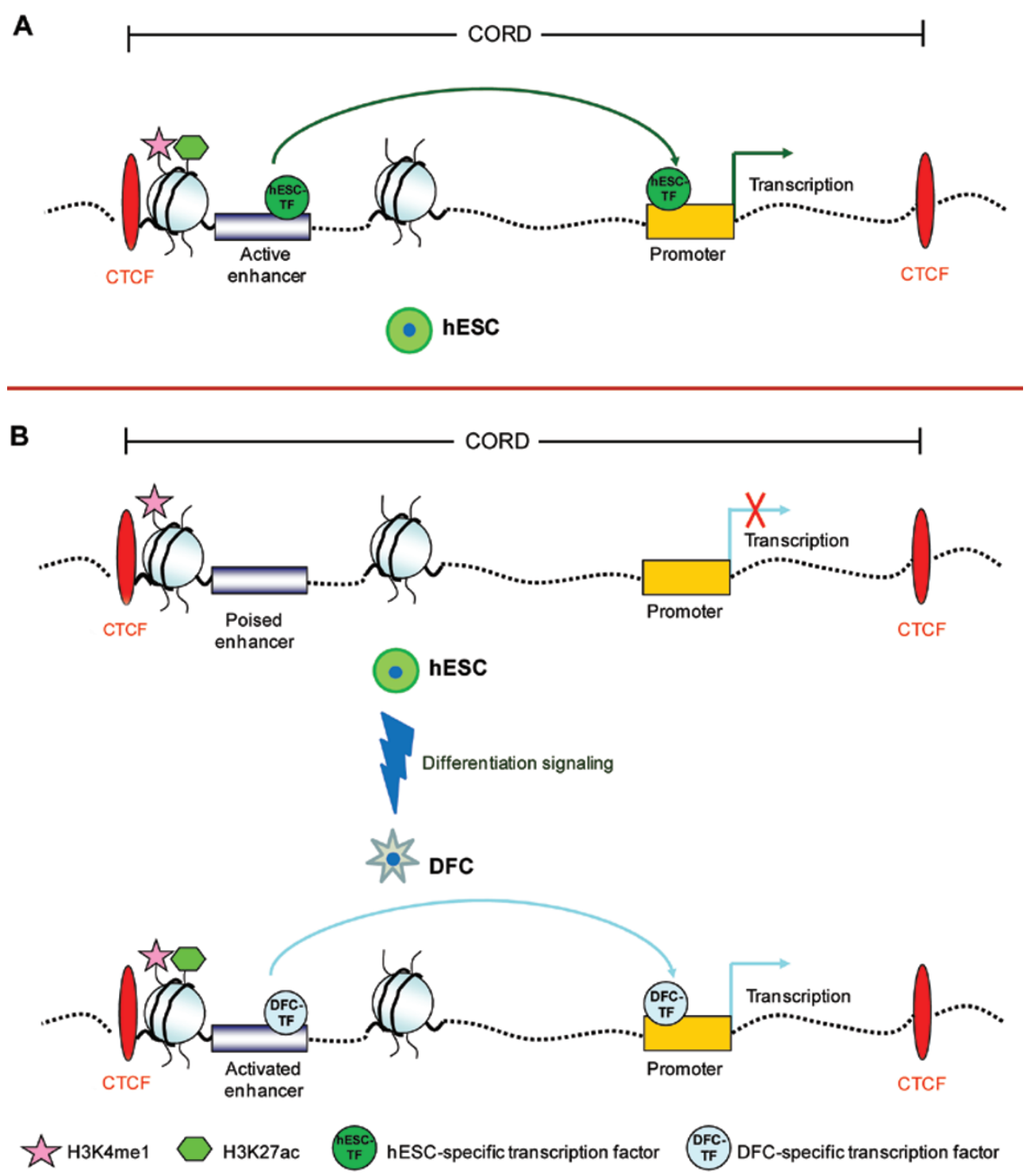

Figure 1 Dynamic chromatin states in pluripotency and differentiation. (A) In undifferentiated hESCs, hESC-specific enhancers are marked by histone H3K4me1 and H3K27ac. Long-range chromatin interaction between the enhancer and the corresponding promoter within a CORD is mediated by hESC-specific transcription factor. The hESC-specific gene is expressed. (B) In DFCs, poised enhancers gain H3K27ac mark. Long-range chromatin interaction between the enhancer and the corresponding promoter within a CORD is mediated by DFC-specific transcription factor. This allows DFC-specific genes to be activated when hESCs are induced to differentiate.

dicted transcription factor binding sites (TFBS) in these enhancers. They found hESC-specific motifs that can be recognized by KLF4, cMYC and FOXD3 and a joint OCT4:SOX2 [6]. This suggests that the putative enhancers are regulated by these transcription factors with known and implicated functions in the maintenance of pluripotency. Remarkably, DFCs-specific motifs are TFBS of transcription factors that regulate early development. Indeed, NANOG and SOX2 ChIP-seq experiments confirmed that these two pluripotency factors are predominantly enriched in hESCsspecific enhancers but not DFCs-specific enhancers. These results imply that other pluripotency-related factors are preferentially located at hESCs-specific enhancers while developmental factors bind to DFCs-specific enhancers when activated. Interestingly, the gain of $\mathrm{H} 3 \mathrm{~K} 4 \mathrm{me} 1$ and $\mathrm{H} 3 \mathrm{~K} 27 \mathrm{ac}$ in enhancers is associated with upregulated genes. Collectively, the authors suggest that both TF binding and dynamic histone modification in enhancers occur in a cell-specific manner.

It has been shown that the chromatin insulator CTCF can mediate long-range 
chromatin interactions between enhancers and their corresponding promoters in murine ES cells [7]. How do these predicted cell-specific enhancers regulate gene expression in hESCs? To address this question, the authors first mapped the binding sites of CTCF in hESCs. They then partitioned the genome into CTCF-organizer regulatory domains (CORDs). From these partitioned CORDs, the authors discovered that hESCs-specific enhancers are highly enriched within the CORDs containing 1000 most hESC-specific genes. On the contrary, the CORDs containing 1000 most DFC upregulated genes are located in DFCs-specific enhancer-enriched CORDs. These suggest that enhancers function in cell-specific manner within CORDs.

Can these predicted enhancers regulate gene expression? The authors cloned 17 predicted hESC-specific enhancers into a luciferase reporter plasmid. The luciferase activity in the majority of these reporter constructs showed high levels of hESC-specific enhancer activity while DFC-specific enhancers did not show activity in hESCs. How do these distal enhancers crosstalk with proximal promoters? The authors conducted chromatin conformation capture (3C) experiments to assay the interaction between 3 enhancers (E1, $\mathrm{E} 2$ and E3) and promoter of FOXD3 in the same CORD. They found that E2, which coincidently showed the highest luciferase activity, had the strongest interaction with the FOXD3 promoter. The authors then extended their prediction of enhancer-promoter interactions to other hESC-specific genes and DFCspecific genes.

Besides hESC-specific enhancers and DFC-specific enhancers, there is a large group of H3K4me1-marked enhancers (nearly 9000 ) that are shared by hESCs and DFCs. To examine the functions of these enhancers, the authors investigated the chromatin changes before and after differentiation. The authors found that many enhancers enriched within CORDs containing DFC-specific genes gain $\mathrm{H} 3 \mathrm{~K} 27 \mathrm{ac}$ but are only marked by $\mathrm{H} 3 \mathrm{~K} 4 \mathrm{me} 1$ in hESCs. Further studies showed that genes located in these CORDs are early differentiation-responsive genes. For example, enhancers of MSX1 and MEIS1 (and many other additional factors) are marked by $\mathrm{H} 3 \mathrm{~K} 4 \mathrm{me} 1$ in both hESCs and DFCs. However, the enhancers gained H3K27ac after the ES cells were induced to differentiate, while $\mathrm{H} 3 \mathrm{~K} 4 \mathrm{me} 1$ was invariant. As such, the gain of $\mathrm{H} 3 \mathrm{~K} 27 \mathrm{ac}$ might be the hallmark of poised enhancers. These H3K27ac-marked poised enhancers may be responsible for early responses in ES cell differentiation.

This important finding is consistent with two other independent studies. Creyghton et al. identified over 25000 H3K4me1-marked enhancers, among which H3K27ac distinguished active enhancers from inactive ones [8]. As such, when mES cells were induced to differentiate into neural progenitor cells (NPC), H3K27ac was gained in only NPC-specific enhancers. In another study, Rada-Iglesias et al. characterized two classes of distal regulatory elements in hESCs [9]. They discovered that class I elements are marked by p300, H3K4me1 and H3K27ac while class II elements are marked by $\mathrm{p} 300$, H3K4me1 and H3K27me3 [9]. Class I-associated genes are highly expressed in hESCs while class II-associated genes are poised for expression [9]. When ES cells were induced to enter the neuroectoderm lineage, a subset of class II genes associated with neuroectoderm gained H3K27ac [9]. Taken together, these studies indicate that $\mathrm{H} 3 \mathrm{~K} 27 \mathrm{ac}$ marks the transition of poised enhancer-associated genes from silencing to activation. Hawkins et al. further unraveled the molecular mechanism behind these observations by conducting reporter assays and $3 \mathrm{C}$ experiments (Figure 1). Future studies could focus on the molecular mechanism behind the rapid switch between
H3K27me3 and H3K27ac. It will also be intriguing to address how transcription factors, chromatin modifiers and extrinsic signaling work together in cell fate decision. Lastly, it would be interesting to determine if the TFs and signaling molecules, which are associated with the H3K27me3/H3K27ac switch, are important in maintaining hESCs identity. Nonetheless, Thomson, Ren and colleagues have provided novel insights into the chromatin landscape of hESCs (Figure 1).

\section{References}

1 Thomson JA, Itskovitz-Eldor J, Shapiro SS, et al. Embryonic stem cell lines derived from human blastocysts. Science 1998; 282:1145-1147.

2 Young RA. Control of the embryonic stem cell state. Cell 2011; 144:940954.

$3 \mathrm{Ng} \mathrm{HH}$, Surani MA. The transcriptional and signalling networks of pluripotency. Nat Cell Biol 2011; 13:490-496.

4 Zhou VW, Goren A, Bernstein BE. Charting histone modifications and the functional organization of mammalian genomes. Nat Rev Genet 2011; 12:718.

5 Hawkins DR, Hon GC, Yang C, et al. Dynamic chromatin states in human ES cells reveal potential regulatory sequences and genes involved in pluripotency. Cell Res 2011; 21:1393-1409.

6 Chen X, Xu H, Yuan P, et al. Integration of external signaling pathways with the core transcriptional network in embryonic stem cells. Cell 2008; 133:1106-1117.

7 Handoko L, Xu H, Li G, et al. CTCFmediated functional chromatin interactome in pluripotent cells. Nat Genet 2011; 43:630-638.

8 Creyghton MP, Cheng AW, Welstead $\mathrm{GG}$, et al. Histone $\mathrm{H} 3 \mathrm{~K} 27 \mathrm{ac}$ separates active from poised enhancers and predicts developmental state. Proc Natl Acad Sci USA 2010; 107:2193121936.

9 Rada-Iglesias A, Bajpai R, Swigut T, et al. A unique chromatin signature uncovers early developmental enhancers in humans. Nature 2011; 470:279-283. 